\title{
Experimental Evaluation of Immediate Recanalization Effect and Recanalization Efficacy of a New Thrombus Retriever for Acute Stroke Treatment In Vivo
}

\author{
P. Mordasini, C. Brekenfeld, J.V. Byrne, U. Fischer, M. Arnold, S. Jung, G. Schroth, and J. Gralla
}

\begin{abstract}
BACKGROUND AND PURPOSE: Currently, several new stent retriever devices for acute stroke treatment are under development and early clinical evaluation. Preclinical testing under standardized conditions is an important first step to evaluate the technical performance and potential of these devices. The aim of this study was to evaluate the immediate recanalization effect, recanalization efficacy, thrombus-device interaction, and safety of a new stent retriever intended for thrombectomy in patients with acute stroke.
\end{abstract}

MATERIAL AND METHODS: The pREset thrombectomy device $(4 \times 20 \mathrm{~mm})$ was evaluated in 16 vessel occlusions in an established swine model. Radiopaque thrombi (10-mm length) were used for visualization of thrombus-device interaction during application and retrieval. Flow-restoration effect immediately after deployment and after 5-minute embedding time before retrieval, recanalization rate after retrieval, thromboembolic events, and complications were assessed. High-resolution FPCT was performed to illustrate thrombus-device interaction during the embedding time.

RESULTS: Immediate flow restoration was achieved in $75 \%$ of occlusions. An increase or stable percentage of recanalizations during embedding time before retrieval was seen in 56.3\%; a decrease, in 12.5\%; reocclusion of a previously recanalized vessel, in $18.8 \%$; and no recanalization effect at all, in 12.5\%. Complete recanalization ( $\mathrm{TICI} 3$ ) after retrieval was achieved in 93.8\%; partial recanalization ( $\mathrm{TICI} 2 \mathrm{~b}$ ), in $6.2 \%$. No distal thromboembolic events were observed. High-resolution FPCT illustrated entrapment of the thrombus between the stent struts and compression against the contralateral vessel wall, leading to partial flow restoration. During retrieval, the thrombus was retained in a straight position within the stent struts.

CONCLUSIONS: In this experimental study, the pREset thrombus retriever showed a high recanalization rate in vivo. High-resolution FPCT allows detailed illustration of the thrombus-device interaction during embedding time and is advocated as an add-on tool to the animal model used in this study.

ABBREVIATIONS: $\mathrm{APA}=$ ascending pharyngeal artery; $\mathrm{FPCT}=$ flat panel $\mathrm{CT} ; \mathrm{IMA}=$ internal maxillary artery; $\mathrm{LA}=$ lingual artery; $\mathrm{TICl}=$ Thrombolysis in Cerebral Infarction

M echanical thrombectomy by using self-expanding retrievable stentlike devices is an emerging treatment approach for acute ischemic stroke. The concept of stent retrievers combines the advantages of temporary stent placement with immediate flow restoration and the advantages of a thrombectomy device with definitive clot removal from the occluded artery. Their potential to reduce time to recanalization and increase recanaliza-

Received February 7, 2012; accepted after revision April 16.

From the Institute of Diagnostic and Interventional Neuroradiology (P.M., C.B., G.S., J.G.) and Department of Neurology (U.F., M.A., S.J.), Inselspital, University of Bern, Bern, Switzerland; and Oxford Neurovascular and Neuroradiology Research Unit (P.M., J.V.B.), Nuffield Department of Surgical Science, The John Radcliffe Hospital, University of Oxford, United Kingdom.

Please address correspondence to Pasquale Mordasini, MD, Department of Diagnostic and Interventional Neuroradiology, Inselspital, University of Bern,

Freiburgstr 4, CH-3010 Bern, Switzerland; e-mail: pasquale.mordasini@insel.ch

http://dx.doi.org/10.3174/ajnr.A3275 tion rates to $>80 \%$ in large cerebral arteries with favorable clinical outcome in a large percentage of patients has been shown in clinical studies. ${ }^{1-5}$

Currently, encouraged by these promising preliminary results, several new stentlike thrombectomy devices for acute stroke treatment are under development and are being introduced into clinical practice. Therefore, preclinical testing of new devices regarding efficacy, thrombus-device interaction, and safety under standardized conditions is important before introduction into clinical routine.

The pREset thrombus retriever (Phenox, Bochum, Germany) is a new stent retriever device designed for thrombectomy in acute stroke treatment. Until now, no experimental or clinical data on its efficacy have been published in the literature, to our knowledge.

The purpose of the present study was to evaluate the recanali- 

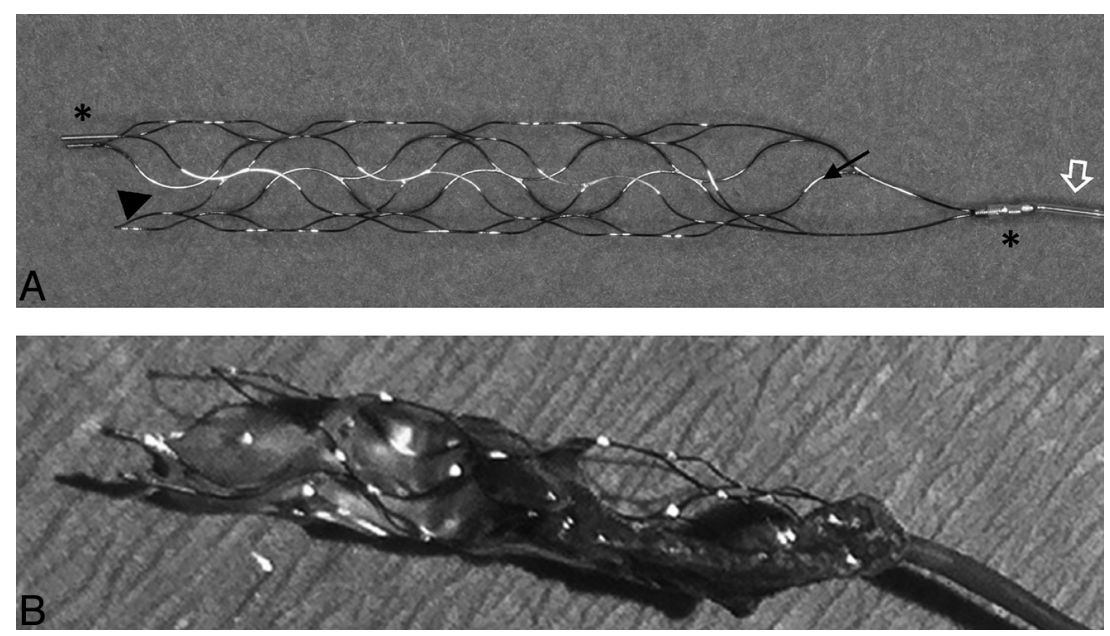

FIG 1. Photograph $(A)$ of the pREset thrombus retriever with an eccentrically attached pusher wire (open arrow) and proximal and distal radiopaque stent markers (asterisks). Note the proximal closed-ring design with a cell connector (arrow) and helically shaped slit in the stent wall (arrowhead). Photograph of the pREset thrombus retriever after a successful thrombectomy procedure $(B)$. Note the engagement of the thrombus by the stent struts and the straight position of the clot along the stent device.

zation rate, immediate recanalization effect, thrombus-device interaction, and safety of the pREset thrombus retriever for acute stroke treatment under standardized experimental conditions in an established animal model of acute vessel occlusion.

\section{MATERIALS AND METHODS}

\section{Thrombectomy Device and Application Procedure}

The pREset thrombus retriever (CE marked in Europe since August 2011) is a laser cut, self-expanding, closed-cell nitinol stent design (Fig 1) with a helically shaped slit within the stent wall. The proximal end of the device consists of a closed-ring design, the cell connector. This closed structure at the proximal portion of the device is intended to create a stable proximal opening, resulting in a constant radial outward force and support. The stent is eccentrically molded onto a $180-\mathrm{cm}$-long pusher wire and is not detachable. The proximal end is marked by 1 and the distal end, by 2 radiopaque markers. The device is available with a diameter of 4 $\mathrm{mm}$ and a length of $20 \mathrm{~mm}$ and is intended for clot retrieval in vessels with diameters of $2-4 \mathrm{~mm}$. It is delivered through a microcatheter with a minimum inner diameter of 0.021 inch and is fully resheathable at any point in the procedure.

In this study, a standard $8 \mathrm{~F}$ balloon-guide catheter for proximal flow arrest during retrieval was used. A 0.021-inch microcatheter (Prowler Select Plus; Cordis, Miami Lakes, Florida) was navigated into the occluded vessel over a 0.014 -inch microwire (SilverSpeed; ev3, Irvine, California), the occlusion site was passed, and the pREset thrombus retriever was inserted. The device was deployed across the thrombus and left in place for an embedding time of 5 minutes, according to previous experimental and clinical experience. ${ }^{4,6}$ Finally, the balloon-guide catheter was inflated to establish flow arrest, and the device and microcatheter were simultaneously retrieved. During retrieval, additional manual aspiration by using a $60-\mathrm{mL}$ lockable syringe was applied at the balloon-guide catheter to avoid embolization of thrombus fragments.

\section{Animal Care}

All procedures were conducted in accordance with international guidelines and were approved by the responsible local authorities. Two 43-kg swine were used in the study. After induction of general anesthesia, a $45-\mathrm{cm}$ long $8 \mathrm{~F}$ catheter sheath (Super Arrow-Flex PSI Set; Teleflex, Limerick, Pennsylvania) was surgically introduced into the common femoral artery and continuously flushed with heparinized saline $(10 \mathrm{IU} / \mathrm{mL})$. No additional heparin was given during the procedures. After the surgical procedure, $500 \mathrm{mg}$ of acetylsalicylic acid was administered intravenously. Further details have been described in previous reports. ${ }^{6-8}$

\section{Thrombus Preparation}

Thrombus preparation and introduction have been described in previous studies in this animal model. ${ }^{6-10}$ In brief, thrombi were generated by mixing $10 \mathrm{~mL}$ of autologous blood with $1 \mathrm{~g}$ of barium sulfate and $0.25 \mathrm{~mL}$ of bovine thrombin solution (Dade thrombin reagent; Siemens Healthcare Diagnostics, Erlangen, Germany). After an incubation time of 1 hour at room temperature, the material was cut into thrombi of 10 -mm length for injection into the target vessel. The resulting embolus was radiopaque and was visible on fluoroscopy during the in vivo procedure.

\section{Experimental Design and Angiographic Evaluation}

Experiments were performed by using a biplane high-resolution angiography system (Axiom Artis zee; Siemens). Selective occlusion of the IMA, the LA, and the APA was performed by injecting the thrombus through a $8 \mathrm{~F}$ balloon-guide catheter positioned proximally in the target vessel. After thrombus injection, the guide catheter was repositioned to restore arterial flow and to allow thrombus embedding for 10 minutes. Immediately after deployment of the device and after an embedding time of 5 minutes, control angiographies were performed to evaluate and measure the effect on flow restoration. Vessel diameters after deployment and embedding time were scored as the percentage of the initial vessel diameter before occlusion. During the embedding time with the device in place, high-resolution FPCT (DynaCT; Siemens) was performed to assess thrombus-device interaction by using the following parameters: 20 -second acquisition, $0.4^{\circ}$ increment, 512 matrix size in projections, $200^{\circ}$ total angle, $10^{\circ}$ per second, and 25 frames per second. Image postprocessing, including multiplanar reconstructions and maximum intensity projection reconstructions as well as 3D volume reconstructions, was performed on a commercially available workstation (Leonardo; Siemens).

Finally, the device was retrieved to evaluate its mechanical thrombectomy efficacy. Only 1 retrieval attempt was allowed per vessel occlusion. Procedure time for each attempt was recorded, including thrombus passage, device deployment, embedding time, and balloon-guide catheter placement and retrieval. All maneuvers under fluoroscopy were monitored and recorded to as- 
sess thrombus movement, fragmentation, and distal dislocation during the passing procedure and device deployment. Angiographies obtained immediately after device deployment and after 5-minute embedding time (ie, before retrieval) were evaluated for thrombus embolization or fragmentation. During the retrieval process, thrombus fragmentation, peripheral embolization, loss of thrombus material at the tip of the balloon-guide catheter, technical feasibility, and technical failure were assessed. Follow-up angiography was performed immediately after retrieval and 1 hour later and was evaluated for the presence of recanalization, downstream emboli, vasospasm, and angiographic signs of vessel dissection or perforation.

\section{Assessment of Vessel Morphology}

The recanalization rate of the target vessel was evaluated by using the TICI grading scale. ${ }^{11}$ Grading of vasospasm was performed according to Grandin et $\mathrm{al}^{12}: 0=$ no narrowing, $1=$ slight narrowing $(<25 \%$ reduction in lumen diameter $), 2=$ moderate narrowing (25\%-50\% stenosis), and $3=$ severe narrowing $(50 \%-$ $75 \%$ stenosis affecting a long segment of the vessel or any stenosis $>75 \%)$.

\section{RESULTS}

A total of 16 retrieval attempts were performed on 16 vessel occlusions $(\mathrm{IMA}=13, \mathrm{LA}=1, \mathrm{APA}=2)$. Mean vessel diameter was $2.6 \pm 0.4 \mathrm{~mm}$ (range, $2.1-3.5 \mathrm{~mm}$ ). In 7 vessels (43.8\%), thrombus lodged at a vessel bifurcation after selective thrombus injection. All injected vessels were totally occluded by the thrombus.

\section{Flow-Restoration Effect}

Immediately after deployment of the pREset device, blood flow was restored in $75.0 \%(12 / 16)$ of vessels. The mean percentage of vessel recanalization was $37.3 \%$ (range, $0 \%-85 \%$ ) immediately after device deployment and 31.8\% (range, 0\%-75.0\%) after 5 -minute embedding time before retrieval. No blood-flow restoration effect, either initially after deployment or after the embedding time, was observed in $12.5 \%$ (2/16). During the 5-minute interval between angiography and retrieval, the percentage of recanalization remained constant or increased in 56.3\% (9/16) of vessels and decreased in $12.5 \%$ (2/16) of vessels; and complete reocclusion occurred in previously partially recanalized vessels in $18.8 \%$ (3/16). Measurements of vessel diameters before vessel occlusion, immediately after stent deployment, and after the embedding time are summarized in the Table.

\section{Retrieval Success}

Complete recanalization (TICI 3) after a single device retrieval was achieved in $93.8 \%$ (15/16). In 1 vessel (6.2\%) with a thrombus lodged at a bifurcation, only partial recanalization (TICI 2b) was accomplished with residual thrombus trapped in the side branch, which was sheared off the device during retrieval; however there was recanalization of the target vessel. In the remaining 6 vessels with thrombus lodged at bifurcations, recanalization of both the target artery and the side branch was achieved. Mean procedure time was 14 minutes (range, 10-23 minutes). Vasospasm rating was grade 0 in $0 \%$, grade 1 in $31.3 \%(5 / 16)$, grade 2 in $50 \%(8 / 16)$, and grade 3 in $18.6 \%(3 / 16)$. Mean vasospasm rating after re-

\begin{tabular}{cccc}
\multicolumn{5}{c}{ Measurements of vessel diameters } \\
$\begin{array}{c}\text { Attempt } \\
\text { No. }\end{array}$ & $\begin{array}{c}\text { Before } \\
\text { Occlusion } \\
(\mathbf{m m})\end{array}$ & $\begin{array}{c}\text { Immediately after } \\
\text { Deployment } \\
(\mathbf{m m})\end{array}$ & $\begin{array}{c}\text { After Embedding } \\
\text { Time }\end{array}$ \\
\hline 1 & 2.7 & 0.8 & 0.9 \\
2 & 2.1 & 1.0 & 1.1 \\
3 & 2.1 & 0.0 & 0.0 \\
4 & 2.3 & 0.0 & 0.4 \\
5 & 2.5 & 1.3 & 1.4 \\
6 & 2.9 & 2.1 & 2.1 \\
7 & 3.0 & 1.2 & 1.1 \\
8 & 3.0 & 0.0 & 0.0 \\
9 & 2.3 & 1.5 & 1.5 \\
10 & 3.5 & 0.8 & 0.0 \\
11 & 2.1 & 0.7 & 0.0 \\
12 & 2.5 & 2.1 & 0.0 \\
13 & 2.9 & 1.8 & 1.9 \\
14 & 3.0 & 1.2 & 1.0 \\
15 & 3.1 & 0.0 & 0.7 \\
16 & 2.3 & 1.1 & 1.2 \\
\hline & & & \\
\hline
\end{tabular}

trieval was 1.9. At follow-up angiography after 1 hour, vasospasms usually had resolved, allowing assessment of the recanalization result.

\section{Thrombus-Device Interaction}

Passage of the occlusion site with the microcatheter was possible in all vessels and always occurred between the thrombus and the vessel wall. No thrombus fragmentation or distal thromboembolic events of the contrasted clot were observed during this maneuver. Unsheathing and deployment of the pREset device across the occlusion site was feasible in all cases.

Assessment of thrombus-device interaction immediately after deployment and after 5-minute embedding time revealed compression of the thrombus against the contralateral vessel wall. At the latter time, expansion of the device allowed partial recanalization of the target vessel in $75 \%(12 / 16)$ of cases, consistent with previous experimental and clinical studies. ${ }^{4,6,8,13}$

Due to the radiopacity of thrombus gained by the added barium sulfate, thrombus-device interaction was shown in detail on the imaging with high-resolution FPCT. 3D volume imaging as well as multiplanar and maximum intensity projection reconstructions of the FPCT raw data showed the thrombus incorporated within the stent struts. The clot predominantly remained within the stent lumen and, to a lesser extent, between the vessel wall and device outer surface. After device deployment, the clot was kept in a straight position along the axis of the vessel and no fragmentation was observed (Figs 2 and 3). During retrieval, thrombus remained embedded within the stent struts in a stable straight position. No significant thrombus compression or elongation was observed during the retrieval. In vessels in which thrombi were situated at bifurcations, clot was extracted in continuity with the thrombus that had extended into side branches, except in 1 vessel as described above (Fig 4). Retrieval of the device into the guide catheter during flow arrest by using proximal balloon occlusion and manual aspiration was uneventful in all cases and showed no shearing of thrombus material when retrieved into the guide-catheter tip. 

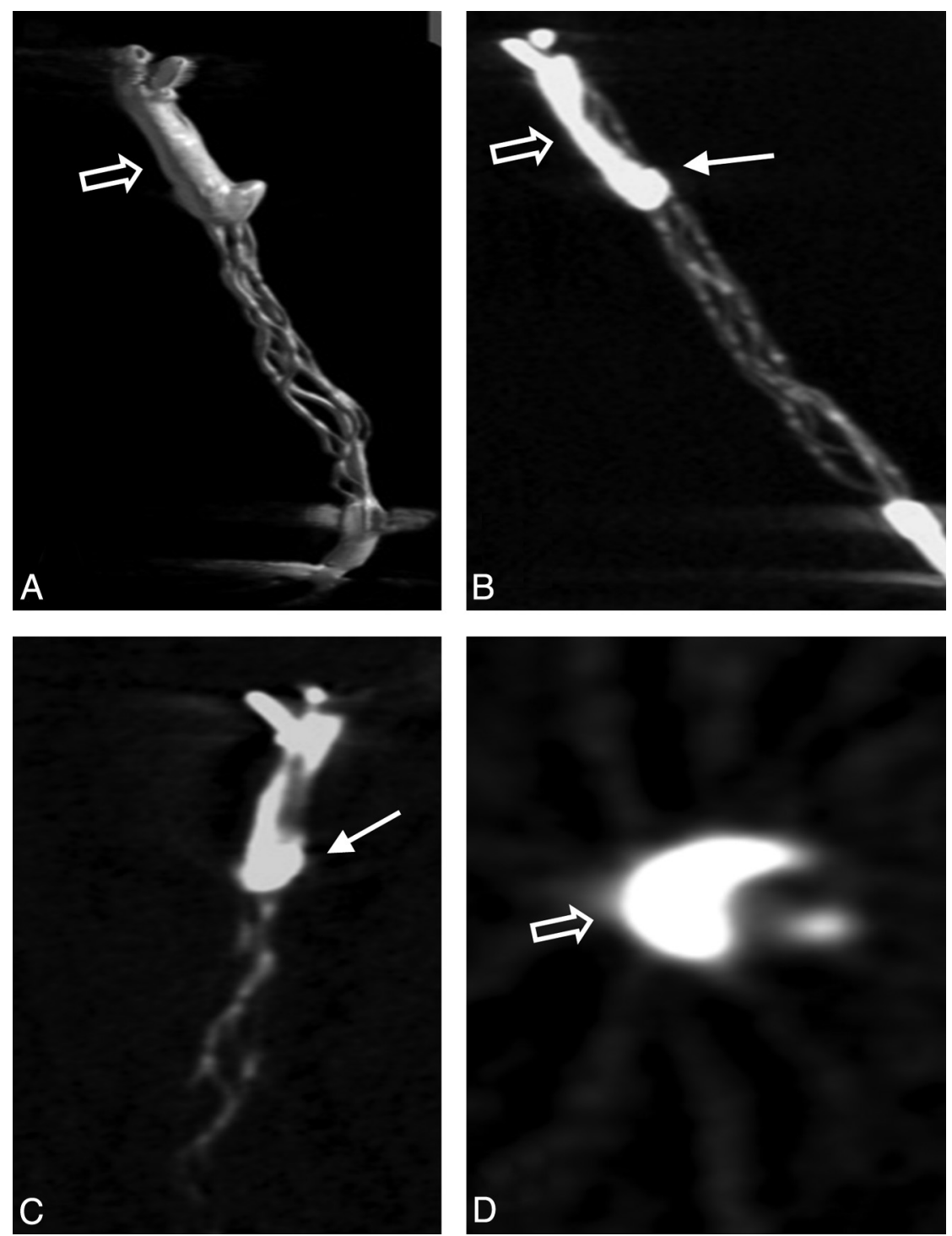

FIG 2. 3D volume reconstruction $(A)$, maximum intensity projection reconstruction $(B)$, and multiplanar reconstructions ( $C$ and $D$ ) of the FPCT. Note the straight position of the opacified thrombus within the stent struts with thrombus portions predominantly inside (arrow) and outside (open arrow) the stent struts.

\section{Complications}

No distal thromboembolic events in the target vessel or previously unaffected vessel territories were observed. No angiographic signs of vessel perforation or dissection were found during control angiography. There was no device fracture or failure during the study.

\section{DISCUSSION}

Mechanical thrombectomy by using self-expandable fully retrievable stentlike thrombectomy devices, so-called stent retrievers, is an emerging treatment option for acute ischemic stroke. Clinical studies have shown that these significantly reduce time to recanalization and improve recanalization rates compared with treatment by intra-arterial thrombolysis and/or other mechanical thrombectomy methods, ${ }^{1-5}$ indicating the potential of this principle to be established as a major approach for endovascular stroke treatment. Because these early promising results are encouraging, several new stent retriever thrombectomy devices with different designs are under development and are continuously added to the armamentarium of endovascular stroke treatment. Therefore, preclinical in vivo testing and evaluation of the efficacy, thrombus-device interaction, and safety of new designs under standardized conditions are recommended before introduction into clinical routine.

Three experimental studies evaluating 2 different stent retrievers in swine and canine models have been published in the literature. ${ }^{6,14,15}$ Jahan $^{14}$ presented the first in vivo assessment of a stent retriever by using the Solitaire FR Revascularization Device (ev3). Complete recanalization (Thrombolysis in Myocardial Infarction 3) was achieved in all 6 of 6 thromboembolic occlusions of the ascending pharyngeal artery in the swine without thromboembolic complications. Another study ${ }^{6}$ by using the swine model and experimental conditions as in the present study also evaluated the Solitaire FR device and reported a complete recanalization rate (TICI 3) of $86.7 \%$ in 13 of 15 vessel occlusions. In 2 cases (13.3\%), partial recanalization (TICI 2b) was achieved with residual thrombus in a ide branch. No thromboembolic events were observed. A more recent study evaluating the Trevo device (Concentric Medical, Mountain View, California) ${ }^{15}$ in swine and canine models of arterial thrombo-occlusive disease showed an equally high recanalization success (100\% Thrombolysis in Myocardial Infarction 3) with extraction of 15/16 thrombi with 1 retrieval attempt and with 2 retrieval attempts in the remaining thrombus. No thromboembolic complications were reported. In the present study, the pREset thrombus retriever was found to be effective for mechanical thrombectomy with a complete recanalization rate (TICI 3 ) of $93.8 \%$ and partial recanalization (TICI $2 \mathrm{~b}$ ) of $6.2 \%$ after a single retrieval attempt. No signs of thromboembolic events or other complications were observed. Overall, recanalization success of the pREset thrombus retriever compares with the recanalization results of the 2 previously described stent retriever systems.

An immediate flow-restoration effect after device deployment was demonstrated in $75 \%$ of cases in the current study, which is consistent with the 50\%-100\% immediate flow-restoration effect reported in the above-mentioned studies. Only 1 study ${ }^{6}$ has so far addressed the flow-restoration effect with time, and this demonstrated a decrease in the probability of progressive recanalization with time, especially after 5 minutes of embedding time. The present study by using the pREset thrombus retriever showed similar findings, in which a stable or increasing percentage of recanalization, compared with the initial vessel diameter, occurred in ap- 

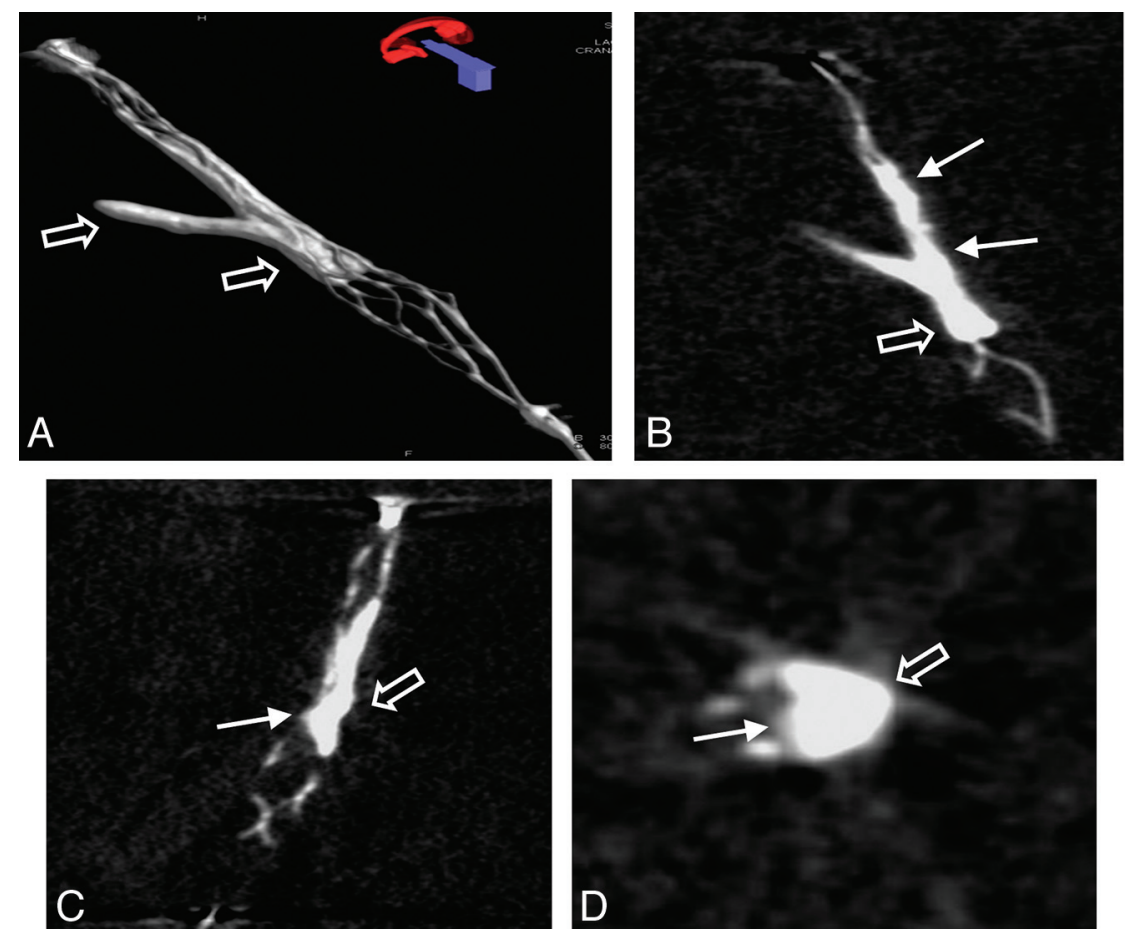

FIG 3. 3D volume reconstruction $(A)$, maximum intensity projection reconstruction $(B)$, and multiplanar reconstructions $(C$ and $D)$ of the FPCT of a thrombus lodging at a vessel bifurcation. Note the typical appearance of the opacified thrombus with thrombus material inside (arrow) and outside (open arrow) in relation to the stent struts.
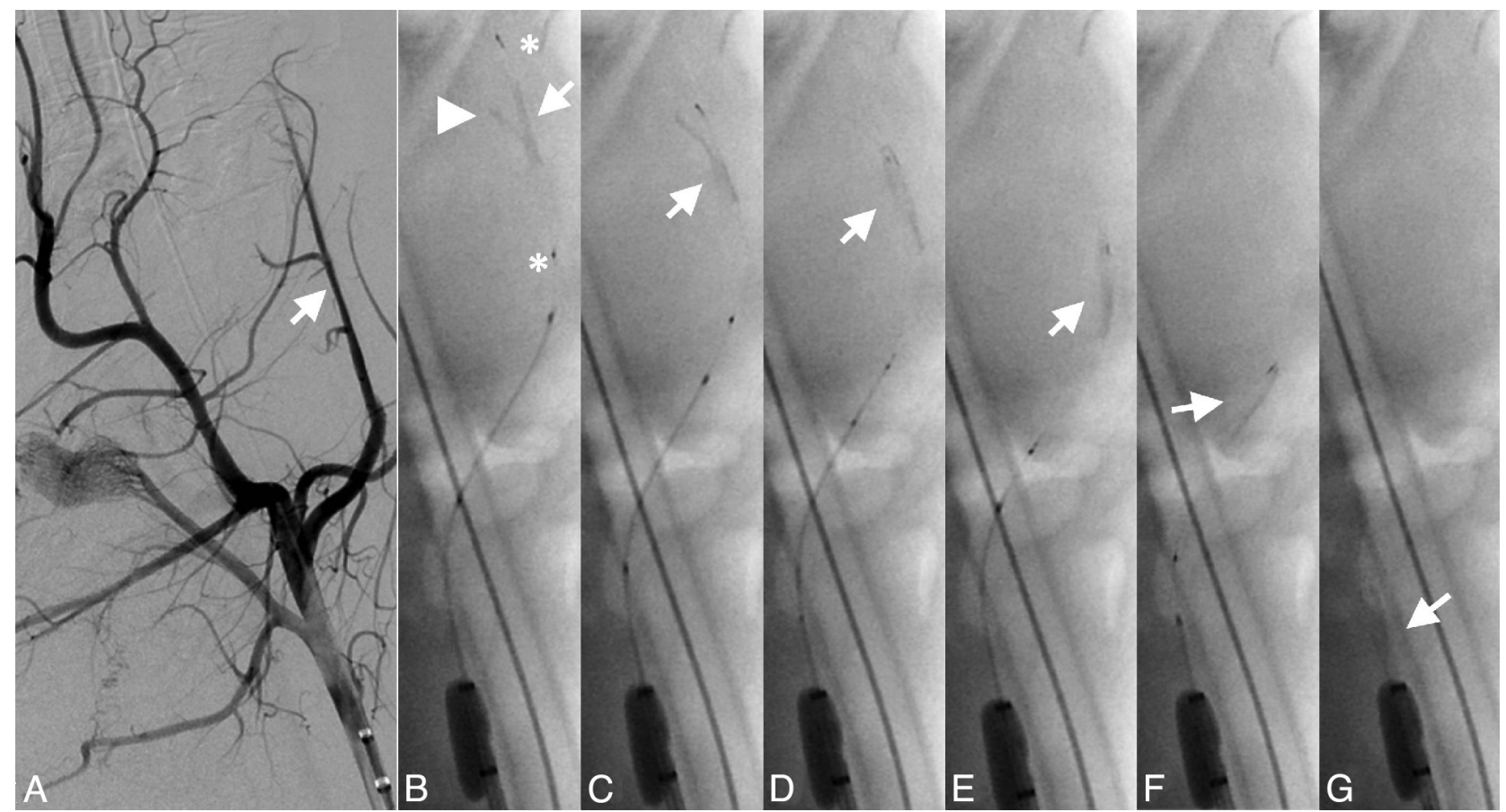

FIG 4. Successful retrieval attempt of a bifurcation thrombus from the LA. Angiogram of the LA (arrow) before occlusion (A) with the main thrombus portion (arrow), side branch portion (arrowhead), and proximal and distal device markers (asterisks) (B). Note retrieval of the side branch portion and the straight position of the thrombus (arrow) during mobilization and retrieval of the device without significant thrombus compression (C-G).

proximately half of the vessels. The remaining vessels showed a decrease in the percentage of recanalization, reocclusion, or no recanalization at all within 5 minutes of embedding time. These observations support the proposition that it is unnecessary to leave a stent retriever in place for $>5$ minutes.
An analysis of thrombus-device interaction evident on highresolution FPCT showed this technique to be a valuable complementary imaging method to characterize thrombus-device interaction in this animal model of acute vessel occlusion. 3D volume, multiplanar, and maximum intensity projection reconstructions 
of the thrombus-device complex enabled a detailed assessment of the position of the thrombus in relation to the stent device and allowed assessment of the principle of action of the device under static conditions during embedding time (Figs 2 and 3).

During mobilization and retrieval of the device, the thrombusdevice complex remained in a straight position without obvious compression or elongation of the clot material. Moreover, firm clot engagement through the stent struts enabled retrieval of thrombi, which had extended into a bifurcation branch (Fig 4). On the other hand, distal thrombectomy devices, which apply a mechanical force to the distal surface of the thrombus (eg, Merci retriever; Concentric Medical), typically lead to compression of the thrombus during extraction, which potentially increases friction between the vessel wall and thrombus. This results in an increased retrieval force needed to mobilize the thrombus and less retrieval success. ${ }^{9,10,16}$ Therefore, straight thrombus position and firm clot engagement seem to be a key feature of stent retrievers compared with other thrombectomy devices and may explain the higher success rates.

\section{Limitations}

The animal model used has been established in several experimental studies evaluating different mechanical treatment approaches for acute stroke treatment. ${ }^{6-10,14-17}$ In our experience, it provides valuable insights into the principle of action of different thrombectomy approaches. However, the model has its inherent shortcomings. First, the vessel anatomy in the swine is less tortuous, less elongated, lacks atherosclerotic changes of the arterial wall, and is more prone to vasospasm compared with the arterial anatomy usually found in human patients with stroke.

Second, the thrombus generated in this model is a wholeblood thrombus that conforms to a slightly firm clot. This type of thrombus might not necessarily be consistent with thrombi found in human patients with stroke. ${ }^{18}$ Furthermore, different thrombus compositions have different mechanical properties and may well influence the thrombus-device interaction, especially its compressibility by a stent device, thrombus stability, and, therefore, the success rate of the thrombectomy procedure.

Finally, assessment of vessel wall dissection or perforation was confined to angiographic evaluation. Therefore, the possible extent of endothelial damage caused by mechanical injury of the vessel wall by retrieving the device could not be fully identified without histologic analysis.

\section{CONCLUSIONS}

This in vivo study of the pREset thrombus retriever showed a high recanalization rate, which compares with the results of other stent retriever devices reported in previous animal studies. In addition, high-resolution FPCT allows a detailed illustration of the thrombus-device interaction during embedding time and is advocated as an add-on tool to the animal model.

Disclosures: James Byrne_UNRELATED: Board Membership: Codman Neurovascular and previously Micrus, Grants/Grants Pending: Siemens AG project grant, * Payment for Lectures (including service on Speakers Bureaus): Boston Scientific and Stryker for teaching.* Marcel Arnold_UNRELATED: Grants/Grants Pending: Swiss National Science Foundation, ${ }^{\star}$ Swiss Heart Foundation, ${ }^{*}$ Shire Human Genetic Therapies.* Gerhard Schroth—RELATED: Grant: ev3," Comments: Study of Tamoxifen and Raloxifene Trial (STAR) Trial (Solitaire stent retriever). Jan Gralla-RELATED: Other: Phenox, Comments: We received the study device for free from the company (Phenox). The study was investigator-driven, and the company has not had access to the manuscript so far, UNRELATED: Consultancy: Covidien, ev3, Comments: Global principal investigator for the STAR Trial (Solitaire FR in acute stroke) and therefore consultant for ev3 completely independent of this study, Grants/Grants Pending: Swiss National Research Foundation, ${ }^{*}$ Travel/Accommodations/Meeting Expenses Unrelated to Activities Listed: Covidien, ev3, Comments: I am the global principal investigator for the STAR Trial (use of Solitaire FR in stroke) and therefore consultant for ev3, completely independent of this study. *Money paid to the institution.

\section{REFERENCES}

1. Castaño C, Dorado L, Guerrero C, et al. Mechanical thrombectomy with the Solitaire $\mathrm{AB}$ device in large artery occlusions of the anterior circulation: a pilot study. Stroke 2010;41:1836-40

2. Roth C, Papanagiotou P, Behnke S, et al. Stent-assisted mechanical recanalization for treatment of acute intracerebral artery occlusions. Stroke 2010;41:2559-67

3. Costalat V, Machi P, Lobotesis K, et al. Rescue, combined, and standalone thrombectomy in the management of large vessel occlusion stroke using the Solitaire device: a prospective 50-patient singlecenter study: timing, safety, and efficacy. Stroke 2011;42:1929-35

4. Brekenfeld C, Schroth G, Mordasini P, et al. Impact of retrievable stents on acute ischemic stroke treatment. AJNR Am J Neuroradiol 2011;32:1269-73

5. Rohde S, Haehnel S, Herweh C, et al. Mechanical thrombectomy in acute embolic stroke: preliminary results with the Revive device. Stroke 2011;42:2954-56

6. Mordasini P, Frabetti N, Gralla J, et al. In vivo evaluation of the first dedicated combined flow-restoration and mechanical thrombectomy device in a swine model of acute vessel occlusion. AJNR Am J Neuroradiol 2011;32:294-300

7. Gralla J, Schroth G, Remonda L, et al. A dedicated animal model for mechanical thrombectomy in acute stroke. AJNR Am J Neuroradiol 2006;27:1357-61

8. Brekenfeld C, Tinguely P, Schroth G, et al. Percutaneous transluminal angioplasty and stent placement in acute vessel occlusion: evaluation of new methods for interventional stroke treatment. AJNR Am J Neuroradiol 2009;30:1165-72

9. Brekenfeld C, Schroth G, El-Koussy M, et al. Mechanical thromboembolectomy for acute ischemic stroke: comparison of the catch thrombectomy device and the Merci Retriever in vivo. Stroke 2008;39:1213-19

10. Mordasini $P$, Hiller M, Brekenfeld $C$, et al. In vivo evaluation of the Phenox CRC mechanical thrombectomy device in a swine model of acute vessel occlusion. AJNR Am J Neuroradiol 2010;31:972-78

11. Higashida RT, Furlan AJ, Roberts $H$, et al. Trial design and reporting standards for intra-arterial cerebral thrombolysis for acute ischemic stroke. Stroke 2003;34:e109-137

12. Grandin CB, Cosnard G, Hammer F, et al. Vasospasm after subarachnoid hemorrhage: diagnosis with MR angiography. AJNR Am J Neuroradiol 2000;21:1611-17

13. Brekenfeld C, Schroth G, Mattle HP, et al. Stent placement in acute cerebral artery occlusion: use of a self-expandable intracranial stent for acute stroke treatment. Stroke 2009;40:847-52

14. Jahan R. Solitaire flow-restoration device for treatment of acute ischemic stroke: safety and recanalization efficacy study in a swine vessel occlusion model. AJNR Am J Neuroradiol 2010;31:1938-43

15. Nogueira RG, Levy EI, Gounis M, et al. The Trevo device: preclinical data of a novel stroke thrombectomy device in two different animal models of arterial thrombo-occlusive disease. J Neurointerv Surg 2012;4:295-300

16. Gralla J, Schroth G, Remonda L, et al. Mechanical thrombectomy for acute ischemic stroke: thrombus-device interaction, efficiency, and complications in vivo. Stroke 2006;37:3019-24

17. Gralla J, Burkhardt M, Schroth G, et al. Occlusion length is a crucial determinant of efficiency and complication rate in thrombectomy for acute ischemic stroke. AJNR Am J Neuroradiol 2008;29:247-52

18. Marder VJ, Chute DJ, Starkman S, et al. Analysis of thrombi retrieved from cerebral arteries of patients with acute ischemic stroke. Stroke 2006;37:2086-93 